Research Paper

\title{
Berberine Reverses Hypoxia-induced Chemoresistance in Breast Cancer through the Inhibition of AMPK- HIF-1a
}

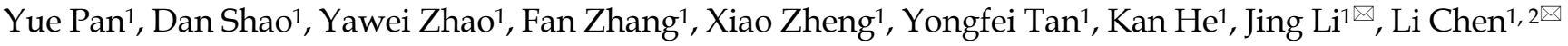 \\ 1. Department of Pharmacology, College of Basic Medical Sciences, Jilin University, Changchun 130021, China; \\ 2. School of Nursing, Jilin University, Changchun 130020, China. \\ $\square$ Corresponding authors: Dr. Li Chen, Department of Pharmacology, Basic College of Medicine, Jilin University, 126 Xinmin Street, Changchun 130021 , \\ People's Republic of China; E-mail: chenl@jlu.edu.cn Telephone/fax: +86 431 85619366. Dr. Jing Li, Department of Pharmacology, Basic College of Medicine, Jilin \\ University, 126 Xinmin Street, Changchun 130021, People's Republic of China; E-mail: lijing@jlu.edu.cn Telephone/fax: +86 43185619799. \\ (c) Ivyspring International Publisher. This is an open access article distributed under the terms of the Creative Commons Attribution (CC BY-NC) license \\ (https:// creativecommons.org/licenses/by-nc/4.0/). See http://ivyspring.com/terms for full terms and conditions.
}

Received: 2016.12.29; Accepted: 2017.04.14; Published: 2017.06.01

\begin{abstract}
Breast cancer is the most common type of cancer and the second leading cause of cancer death in American women. Chemoresistance is common and inevitable after a variable period of time. Therefore, chemosensitization is a necessary strategy on drug-resistant breast cancer. In this study, MCF-7 breast cancer cell was cultured under hypoxia for a week to induce the resistance to doxorubincin (DOX). The effect of different doses of berberine, a traditional Chinese medicine, on DOX sensitivity to MFC-7/hypoxia cells was observed. We found that hypoxia increased DOX resistance on breast cancer cells with the AMPK activation. Low-dose berberine could resensitize DOX chemosensitivity in MCF-7/hypoxia cell, however, high-dose berberine directly induced apoptosis. The intriguing fact was that the protein expressions of AMPK and HIF-l $\alpha$ were down-regulated by berberine, either low dose or high dose. But the downstream of HIF-la occurred the bifurcation dependent on the dosage of berberine: AMPK-HIF-la-P-gP inactivation played a crucial role on the DOX chemosensitivity of low-dose berberine, while AMPK-HIF-1 $\alpha$ downregulaton inducing $\mathrm{p} 53$ activation led to apoptosis in high-dose berberine. These results were consistent to the transplanted mice model bearing MCF-7 drug-resistance tumor treated by berberine combined with DOX or high-dose berberine alone. This work shed light on a potentially therapeutic attempt to overcome drug-resistant breast cancer.
\end{abstract}

Key words: berberine; hypoxia; breast cancer resistance; AMPK; HIF-1a.

\section{Introduction}

Breast cancer is the most common type of cancer and the second leading cause of cancer death in American women. In 2017, 252,710 estimate new cases and 40,610 estimate deaths are projected to occur in the United States [1]. Surgery, radiation therapy, systemic treatment and personalized medicine are the main managements for breast cancer. Adjuvant chemotherapy in systemic treatment for breast cancer is popular in China, with about $81.4 \%$ of all patients with invasive breast cancer starting chemotherapy [2]. Generally, systemic agents are effective at the beginning of therapy in $90 \%$ of primary breast cancers and $50 \%$ of metastases. However, drug resistance to therapy is not only common but expected after a variable period of time. To delay the generation of chemoresistance to increase efficacy, chemosensilization is a necessary strategy based on the breast cancer patient with drug-resistant molecular characteristics.

Hypoxia microenvironment commonly exists in human solid tumors, including breast cancer, lung cancer, oral cancer and ovarian cancer. It also contributes to the tumor multidrug resistance (MDR) [3]. A growing amount of evidence suggests that drug 
extrusion by cell membrane pumps, drug-induced apoptosis inhibition and drug target molecules modification might play crucial roles on hypoxia-induced chemotherapeutic resistance [4]. The transcription factor hypoxia-inducible factor-1 (HIF-1) maintained responses to hypoxic environment and closely related to the proliferation, apoptosis, angiogenesis and drug resistance of cancer $[5,6]$. One of the most prominent hallmarkers of drug resistance is the over-expression of P-glycoprotein (P-gp). Drug resistance induced by hypoxia mediated by up-regulating P-gp protein expression through HIF-1a activating [7]. Apart from the over-expression of P-gp, apoptosis inhibition is another important mechanism for drug resistance [8]. Since p53, a multi-functional transcription factor that suppresses tumor, could regulate cellular proliferation, cell death, mutagenesis, DNA repair and apoptosis by targeting an array of genes, such as MDM2, BAX, DR4 and DR5 etc [9], hence, p53 activation might overcome drug resistance through inducing apoptosis. It is reported that the hypoxia environment promoted HIF-1a expression and escaped from apoptosis by inhibiting p53 [10].

AMP-activated protein kinase (AMPK), the sensor of cellular energy, is activated by hypoxia to compensate the reduced mitochondrial respiration $[11,12]$. AMPK modulating P-gp was involved in hypoxia-induced multidrug resistance in human cancer cells [13]. Berberine (Ber), an isoquinoline alkaloid purified from the Berberis species, has exhibited multiple pharmacological activities, including antibacterial, anti-hypertensive, anti-arrhythmic and antitumor effect [14]. Moreover, it has been showed that berberine overcomed radio-resistance in colon cancer by inhibiting P-gp expression [15]. Berberine also has been reported to downregulate AMPK expression in ischemic areas of rat heart caused by ligating coronary artery [16]. Therefore, we hypothesized that berberine might overcome drug resistance induced by hypoxia, but its molecular mechanisms still need to further explore.

In this study, we firstly set up the drug-resistant cell model using MCF-7 cell under the condition of hypoxia. Secondly, we investigated whether berberine could overcome DOX chemoresistance on MCF-7/hypoxia cell and revealed the relative molecular mechanisms. Thirdly, the transplanted mice model bearing drug-resistant tumor was used to detect whether berberine could resensitilize DOX resistance in vivo. This work is beneficial to attempt a novel strategy for drug-resistance cancer treatment.

\section{Materials and Methods}

\section{Chemicals and reagents}

Sulforhodamine B was purchased from Sigma Aldrich. IOX2 and AICAR were purchased from Selleckchem. DMEM medium, fetal bovine serum (FBS), penicillin, streptomycin and BCA protein assay kits were from Beyotime Institute of Biotechnology (Haimen, China). The primary antibodies were diluted 1:1000 before use, including AMPK (Cat. \# sc-25792, Santa Cruz Biotechnologies), p-AMPK (Cat. \# sc-33524, Santa Cruz Biotechnologies), HIF-1a (Cat. \#113642 Abcam), P-gp (Cat. \# sc-55510, Santa Cruz Biotechnologies), p53 (Cat. \# 10442-1-AP, Proteintech), Bax (Cat. \# sc-7480, Santa Cruz Biotechnologies), Cytochrome c (Cat. \# sc-13561, Santa Cruz Biotechnologies), Caspase 9 (Cat. \# 842, Cell signaling), Caspase 3 (Cat. \# 836, Cell signaling), PARP (Cat. \# 1442, Cell signaling) and $\beta$-actin (Cat. \# sc-130300, Santa Cruz Biotechnologies). All the chemical compounds were analytically pure reagents. Berberine is a gift from Northeast Pharmaceutical Factory. All other chemicals and reagents were of analytical grade.

\section{Cell culture and SRB assay}

MCF-7 cells were maintained in DMEM high glucose medium supplemented with $10 \%$ fetal bovine serum (FBS), 100 units $/ \mathrm{mL}$ penicillin, and $100 \mu \mathrm{g} / \mathrm{mL}$ streptomycin in an atmosphere of $95 \%$ air and $5 \% \mathrm{CO}_{2}$ at $37^{\circ} \mathrm{C}$. For hypoxic exposure, MCF-7 cells were maintained in DMEM high glucose medium supplemented with $10 \%$ fetal bovine serum (FBS), 100 units $/ \mathrm{mL}$ penicillin, and $100 \mu \mathrm{g} / \mathrm{mL}$ streptomycin and cultured in a modulator incubator chamber at $37^{\circ} \mathrm{C}$ with $94 \% \mathrm{~N}_{2}, 1 \% \mathrm{O}_{2}$ and $5 \% \mathrm{CO}_{2}$ for at least 7 days. The sulforhodamine B (SRB) assay is routinely used for cytotoxicity determination. MCF-7 and MCF-7/hypoxia were seeded in 96 well plates at a density of $5 \times 10^{3}$ cells per well overnight, and treated with the indicated with DOX at final concentrations of $0,0.04,0.08,0.16,1.25,2.5,5,10,20$ and $40 \mu \mathrm{g} / \mathrm{mL}$ for $48 \mathrm{~h}$. MCF-7/hypoxia treated with Berberine at final concentrations of $0,1.25,2.5,5,10,20,40,80$ and 160 $\mu \mathrm{M}$ and/or $0.8 \mu \mathrm{M}$ AICAR for $48 \mathrm{~h}$. MCF-7/hypoxia treated with Berberine at final concentrations of 0 , $1.25,2.5,5,10,20,40,80,160 \mu \mathrm{M}$ and/or $6.25 \mu \mathrm{M}$ IOX2 for $48 \mathrm{~h}$. MCF-7/hypoxia were seeded at a density of $5 \times 10^{3}$ cells/well overnight, and treated with DOX at final concentrations of $0,0.04,0.08,0.16,1.25,2.5,5$ $\mu \mathrm{g} / \mathrm{mL}$ and co-treated with $2.5,5,10 \mu \mathrm{M}$ berberine separately. MCF-7/hypoxia treated with different concentrations DOX and co-treated with 2.5, 5, $10 \mu \mathrm{M}$ berberine separately were indicated with/or $0.8 \mu \mathrm{M}$ AICAR for $48 \mathrm{~h}$. MCF-7/hypoxia treated with 
different concentrations DOX and co-treated with 2.5, 5, $10 \mu \mathrm{M}$ berberine separately were indicated with/or $6.25 \mu \mathrm{M}$ IOX2 for $48 \mathrm{~h}$. the cells were fixed with $10 \%$ trichloroacetic acid, and $0.4 \%(\mathrm{w} / \mathrm{v}) \mathrm{SRB}$ in $1 \%$ acetic acid was added to stain the cells. Unbound SRB was washed away with $1 \%$ acetic acid and SRB-bound cells were rendered soluble with $10 \mathrm{mM}$ Tris-base $(\mathrm{pH}$ 10.5; Sigma Aldrich). The absorbance was read at a wavelength of $570 \mathrm{~nm}$.

\section{Apoptosis detection by flow cytometry}

Cell death and apoptosis were detected with an Annexin V-FITC Apoptotic Detection Kit by flow cytometry. After treating with 0,5 and $40 \mu \mathrm{M}$ berberine for $48 \mathrm{~h}, \mathrm{MCF}-7 /$ hypoxia cells were harvested and washed twice with cold PBS. The cell pellets were resuspended with binding buffer to cell suspension at a density of $1 \times 10^{6}$ cells $/ \mathrm{mL}$. Then $5 \mu \mathrm{L}$ of FITC-conjugated annexin $\mathrm{V}$ was added to the suspension and incubated for $15 \mathrm{~min}$ at $4^{\circ} \mathrm{C}$ in the dark. After that, $5 \mu \mathrm{L}$ of propidium iodide (PI) was injected into the mixture and incubated with cells for 5 min. The samples were subsequently analyzed by flow cytometry with an FACS Calibur flow cytometer (BD Biosciences, San Jose, CA, USA). The excitation wavelength was $488 \mathrm{~nm}$, the emission of FITC-conjugated annexin $\mathrm{V}$ was $515 \mathrm{~nm}$ and the propidium iodide (PI) emission was $560 \mathrm{~nm}$.

\section{Western blot}

MCF-7 and MCF-7/hypoxia cells total protein and cytoplasm were collected. MCF-7/hypoxia were grown in berberine with the final concentrations of 0 , 5, $40 \mu \mathrm{M}$ for $48 \mathrm{~h}$, total protein and cytoplasm were collected. MCF-7/ hypoxia were treated with the final concentrations of $0,5,40 \mu \mathrm{M}$ and/or $0.8 \mu \mathrm{M}$ AICAR for $48 \mathrm{~h}$, total protein was collected. MCF-7/hypoxia were treated with the final concentrations of $0,5,40$ $\mu \mathrm{M}$ and and/or $6.25 \mu \mathrm{M}$ IOX2 for $48 \mathrm{~h}$, total protein was collected. Total protein was extracted from tumor tissue or cells were washed with ice-cold PBS and lysed with RIPA Cell Lysis Buffer (Cell Signaling) containing a phosphatase inhibitor and the protease inhibitor cocktail (Sigma) by incubating on ice for 30 min. Lysates were collected by centrifugation and protein concentrations were determined by the BCA method. The samples were separated on $12 \%$ SDS-PAGE and transferred onto polyvinylidene fluoride (PVDF) membranes. After blocking in TBS buffer (150 mM NaCl, $10 \mathrm{mM}$ Tris, $\mathrm{pH} 7.4)$ containing $5 \%$ nonfat milk, the blots were incubated with a primary antibody (rabbit anti AMPK, p-AMPK, HIF-1a, P-gp, p53, Bax, cytochrome c, Caspase 9, Caspase 3 , PARP and $\beta$-actin) at $4{ }^{\circ} \mathrm{C}$ overnight and secondary antibody for $1 \mathrm{~h}$ at room temperature. The corresponding horseradish peroxidase-conjugated secondary antibodies (1:2000 dilutions) were incubated at room temperature. The blots were visualized by super ECL and quantified by software Quantity One (BIO-RAD). $\beta$-actin was used as the internal control.

\section{Subcutaneous transplanted tumor model in nude mice}

Animal experiment protocols were approved by the Ethics Committee for the Use of Experimental Animals of Jilin University. MCF-7/hypoxia $\left(2 \times 10^{6}\right)$ were collected in $70 \mu \mathrm{L}$ PBS and mixed with $70 \mathrm{~mL}$ Matrigel Matrix (Becton Dickinson Biosciences). The mixture was injected subcutaneously on one side of the dorsal flank of 8-week-old female BALB/c nu/nu mice (Vital River Laboratories, Beijing, China). When tumor volume reached $60 \sim 100 \mathrm{~mm}^{3}$, mice were randomized into 5 groups $(n=6)$. The mice in control group treated with saline injected by tail vein every 3 days, the DOX group treated with DOX $\left(1 \mathrm{mg} \mathrm{kg}^{-1}\right)$ by tail vein injection every 3 days, the low dose berberine(Ber-L) group treated with berberine (5 $\mathrm{mg} \mathrm{kg}^{-1}$ ) daily by oral gavage, the low dose berberine combined with DOX group (Ber-L+DOX) treated with berberine $\left(5 \mathrm{mg} \mathrm{kg}^{-1}\right)$ daily by oral gavage and DOX (1 $\mathrm{mg} \mathrm{kg}^{-1}$ ) by tail vein injection every 3 days, the high dose berberine group treated with berberine $\left(200 \mathrm{mg} \mathrm{kg}^{-1}\right)$ daily by oral gavage. The body weights and tumor sizes were accurately recorded every three days, the tumor volume was calculated according to the formula: length $\times \mathrm{Width}^{2} \times 0.52$. The mice were sacrificed 25 days after drug administration. At the end of the study, the tumor tissues were isolated and stored in $-80^{\circ} \mathrm{C}$ immediately for later analysis.

\section{Statistical analysis}

All data were expressed as mean \pm SD. Statistical significances among groups were analyzed by one-way analysis of variance (ANOVA) followed by Dunnett's multiple comparison post. A value with $p<$ 0.05 was considered as statistically significant.

\section{Results}

\section{Hypoxia increases DOX resistance on breast cancer cells with AMPK activation}

To investigate the effect of hypoxia on DOX chemotherapy sensitivity, MCF-7 cells in controlled normoxia environment (MCF-7/normoxia) and MCF-7 cells in controlled hypoxia environment (MCF-7/hypoxia) were relatively exposed to the various concentrations of DOX $(0.04 \sim 40 \mu \mathrm{g} / \mathrm{mL})$ for $48 \mathrm{~h}$. Cell viability was determined by SRB assay. Compared with MCF-7/normoxia, the cell viability in 
MCF-7/hypoxia increases about $8.4 \% \sim 60.1 \%$ at the DOX dosage range from $0.16 \mu \mathrm{g} / \mathrm{mL}$ to $10 \mu \mathrm{g} / \mathrm{mL}$ (Fig. 1A), and hypoxia reduced the DOX intracellular concentration (Fig. S1), indicating that hypoxia induces DOX chemoresistance. To discuss the related drug-resistant proteins in MCF-7 cell under hypoxia, we evaluated AMPK signaling pathway, which is correlated with enzyme metabolism under hypoxia. It is showed that the protein expressions of the phosphorylated AMPK (p-AMPK), HIF-1a and P-gp significantly increased, indicating that hypoxia induces the activation of AMPK (Fig. 1B, S2).

\section{Cytotoxicity effect of berberine on MCF-7/hypoxia cells}

To assess cytotoxicity profile of berberine on MCF-7/hypoxia cells, a series of concentrations of berberine (from $1.25 \sim 160 \mu \mathrm{M}$ ) were used to treat MCF-7/hypoxia cells for $48 \mathrm{~h}$ and $72 \mathrm{~h}$. As shown in Fig. 2A, berberine has negligible influence on cell growth when the concentration is less than $10 \mu \mathrm{M}$. However, when the concentration is more than $20 \mu \mathrm{M}$, berberine inhibits MCF-7/hypoxia cell growth in a time- and dose-dependent manner, and then apoptosis of MCF-7/hypoxia cell treated with $5 \mu \mathrm{M}$ and $40 \mu \mathrm{M}$ berberine for $48 \mathrm{~h}$ was determined by flow cytometry, as shown in Fig. 2B, early apoptotic rate (Q4: $3.1 \pm 0.53 \%$ vs. $2.85 \pm 0.95 \%$ ) and late apoptotic or necrotic rate (Q2: $3.51 \pm 0.79 \%$ vs. $3.71 \pm 0.83 \%)$ in 5 $\mu \mathrm{M}$ berberine have no significant difference compared with control group, but in $40 \mu \mathrm{M}$ berberine treatment group, the early apoptotic rate is $10.67 \pm 1.88 \%$, the late apoptotic or necrotic rate is $28.73 \pm 6.21 \%$, indicating that high dose berberine $(40 \mu \mathrm{M})$ could cause MCF-7/hypoxia cells apoptosis.

Moreover, we detected the protein expressions in AMPK signaling pathway and canonical mitochondrial apoptosis pathway. It is showed that a-week-hypoxia induced p-AMPK, HIF-1a and P-gp protein expressions of MCF-7/hypoxia cell in a stable and high level, in contrast to MCF-7/normoxia (Fig. $1 \mathrm{~B}$ S2). However, berberine decreases the three protein expressions in dose-dependent manner. High-dose berberine significantly activates p53 and mitochondrial apoptosis pathway with a series of proteins releasing, including BAX, Cytochrome c, cleaved-Caspase 9, cleaved-Caspase 3 and cleaved PARP (Fig. 2C, S3). According to this results, we designed using low dose berberine (less than $10 \mu \mathrm{M}$ ) combining with DOX to detect the change of DOX chemosensitivity.

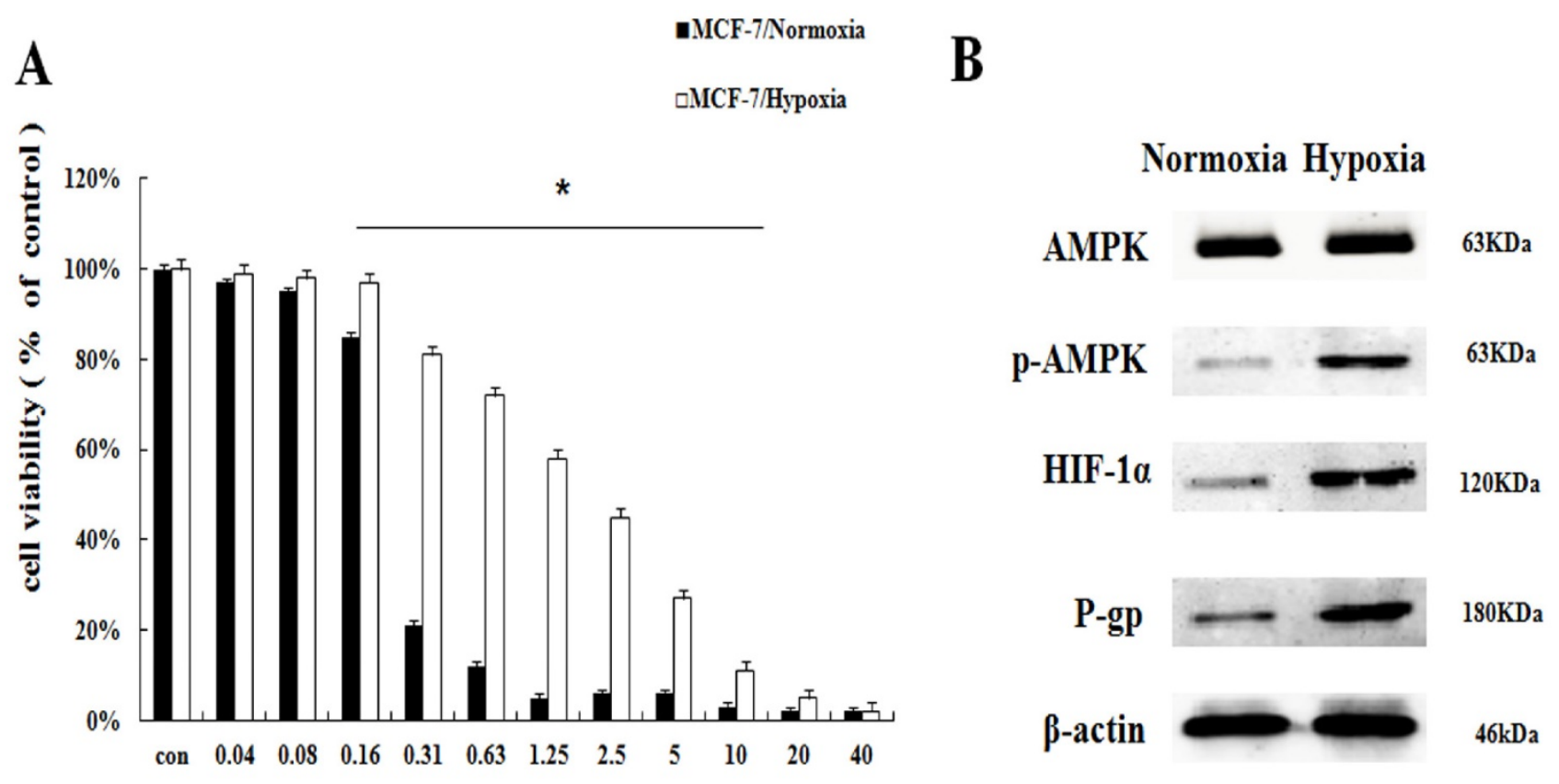

\section{DOX concentration $(\mu \mathrm{g} / \mathrm{ml})$}

Figure 1. Hypoxia reduced the DOX chemoresistance with the AMPK signaling pathway in MCF-7/hypoxia (A) The DOX cytotoxicity effect in MCF-7/normoxia and MCF-7/hypoxia cell. Mean values \pm SD, * P <0.05, MCF-7/normoxia vs. MCF-7/hypoxia (B) AMPK, p-AMPK, HIF-1 $\alpha$ and P-gp proteins expression in MCF-7/normoxia and MCF-7/hypoxia cell. 
A

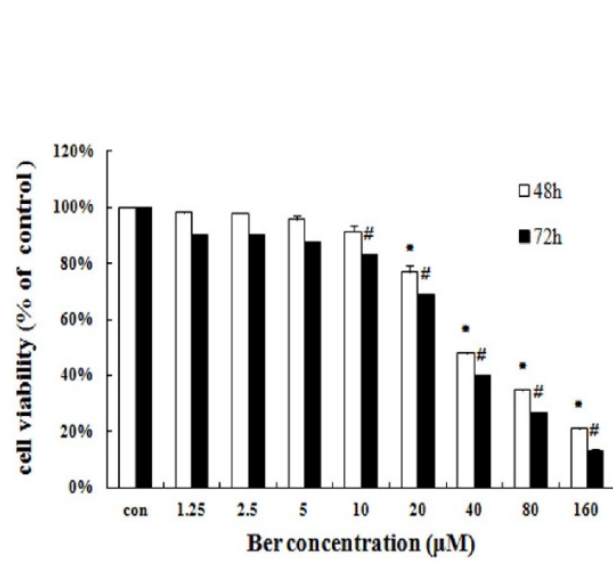

B
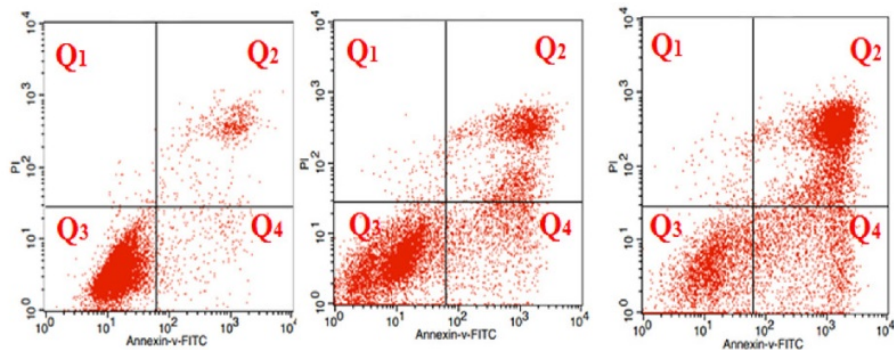

CON

$\operatorname{Ber}(5 \mu \mathrm{M})$

$5 \mu \mathrm{mol} / \mathrm{L}$

$0.32 \pm 0.12$

$2.31 \pm 0.63$

Q2(\%) $\quad 3.71 \pm 0.83$

$3.51 \pm 0.79$

$28.73 \pm 6.21$

Q3(\%) $\quad 92.04 \pm 7.69$

$93.17 \pm 9.21$

$58.29 \pm 9.17$

Q4(\%) $\quad 3.85 \pm 0.95 \quad 3.1 \pm 0.53$

$10.67 \pm 1.88$

Berberine concentration $(\mu \mathrm{M})$

C

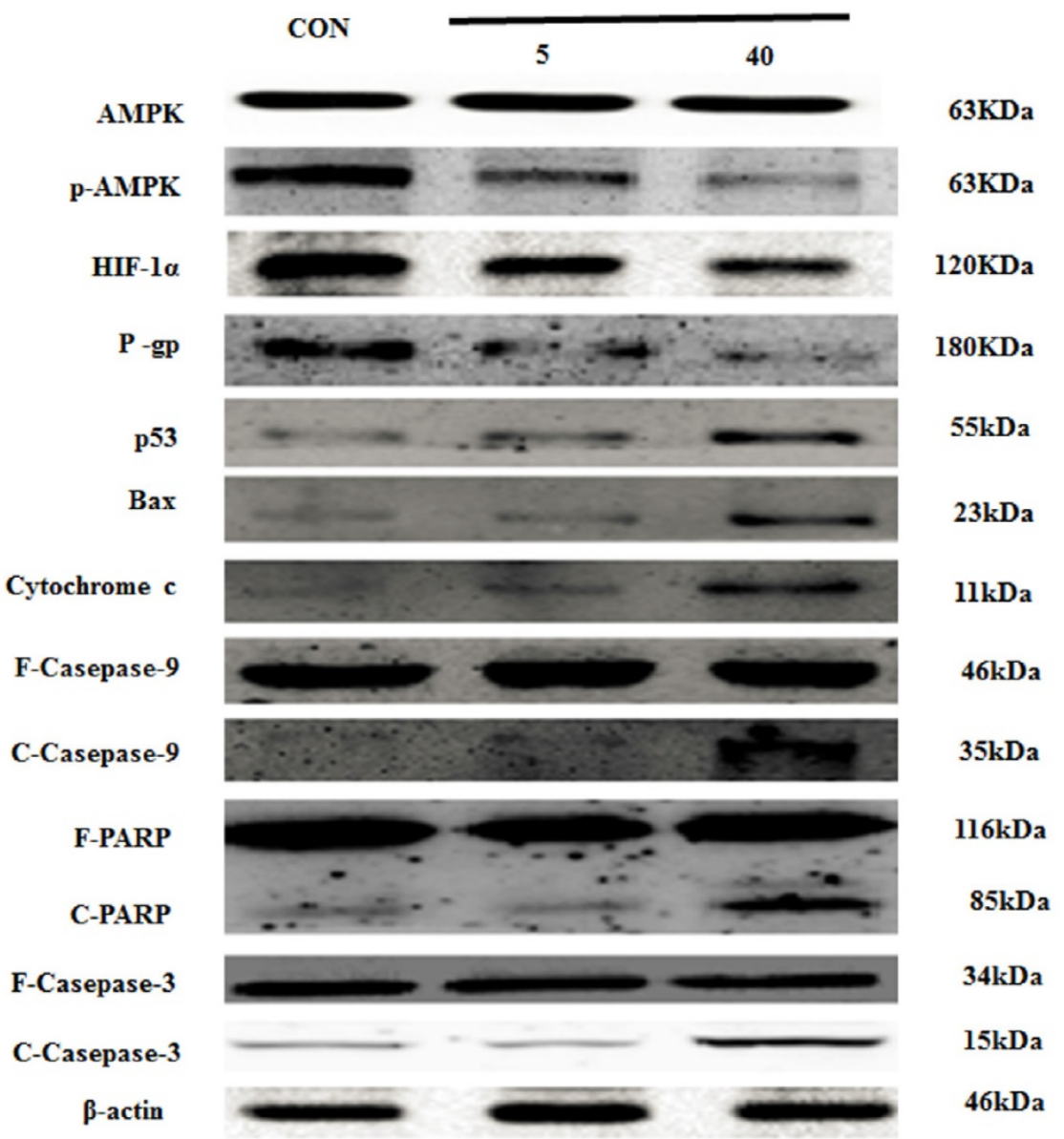

Figure 2. The apoptosis effect in MCF-7/hypoxia caused by berberine (A) The cytotoxicity effect in MCF-7/hypoxia cell caused by berberine for 48 and $72 \mathrm{~h}$. Mean values $\pm S D$, * $P<0.05$, cells treated with different concentrations of berbeine for $48 \mathrm{~h}$ vs. control group. \# $P<0.05$, cells treated with different concentrations of berberine for $72 \mathrm{~h}$ vs. control group. (B) The apoptosis rate in MCF-7/hypoxia caused by 0,5 and $40 \mu \mathrm{M}$ berberine for $48 \mathrm{~h}$. (C) AMPK, p-AMPK, HIF-1 $\mathrm{a}, \mathrm{P}$-gp, $\mathrm{p} 53$, Bax, cytochrome c, Caspase 9, Caspase 3 and PARP proteins expression by 0,5 and $40 \mu M$ berberine treatment for $48 \mathrm{~h}$ in MCF-7/hypoxia. Each result was repeated three times. 


\section{Low-dose berberine resensitized DOX chemosensitivity of MCF-7/hypoxia cells}

To investigate whether low-dose berberine could increase DOX chemosensitivity, we treated MCF-7/hypoxia cells with DOX (concentrations from 0.04 to $5 \mu \mathrm{g} / \mathrm{mL}$ ) for $48 \mathrm{~h}$ and $72 \mathrm{~h}$ combined with low concentrations of berberine $(2.5 \mu \mathrm{M}, 5 \mu \mathrm{M}$ and $10 \mu \mathrm{M})$. As shown in Fig. 3A and 3B, low-dose berberine (2.5 $10 \mu \mathrm{M})$ significantly improves DOX sensitivity on MCF-7/hypoxia cells for $48 \mathrm{~h}$ and $72 \mathrm{~h}$ treatment, IC50 of DOX decreases from $2.48 \mu \mathrm{g} / \mathrm{mL}$ (DOX alone) to $1.37 \mu \mathrm{g} / \mathrm{mL}$ (combined with $2.5 \mu \mathrm{M}$ berberine), $0.49 \mu \mathrm{g} / \mathrm{mL}$ (combined with $5 \mu \mathrm{M}$ berberine) and 0.34 $\mu \mathrm{g} / \mathrm{mL}$ (combined with $10 \mu \mathrm{M}$ berberine) for $48 \mathrm{~h}$ treatment. Giving that a $10 \mu \mathrm{M}$ berberine alone has negligible effect on cell growth of MCF-7/hypoxia cells (Fig. 2A), it indicates that low-dose berberine could resensitize the DOX chemsensitivity to MCF-7/hypoxia cell lines.

\section{Low-dose berberine suppresses P-gp by down-regulating the AMPK-HIF-1 $\alpha$ signaling pathway}

To explore the downstream of AMPK signaling, we used AMPK activator AICAR to find out the downstream target proteins of AMPK. As indicated in Fig. 3A, low-dose berberine $(5 \mu \mathrm{M})$ combined with DOX strongly inhibits MCF- 7/hypoxia cell growth, however, in the presence of AICAR, the inhibition of cell growth is significantly weaken compared with the same concentration of the berberine plus DOX treatment (Fig. 4A). As shown in Fig. 4C and S4,
AICAR up-regulating p-AMPK protein expression, counteracts the inhibition of p-AMPK induced by low-dose berberine. And HIF-1a and P-gp protein expressions increase with p-AMPK up-regulation induced by AICAR, indicating that HIF-1a and P-gp are the downstream of AMPK.

To further explore the relationship between HIF-1a and P-gp, HIF-1a stabilizer IOX2 was used. IOX2 can stabilize HIF-1a by selectively inhibiting prolyl-4-hydroxylase-2 (PHD-2), which can covalently modify two proline residues in the oxygen-dependent degradation (ODD) domain of HIF-1a subunits to promote HIF-1a degradation under hypoxia $[17,18]$. The increase of DOX chemosensitivity induced by berberine in MCF-7/hypoxia cells is weaken by the addition of IOX2 together with up-regulation of P-gp protein expression, and at the same time, the p-AMPK expression do not change (Fig. 5A, 5C and S5). Our result revealed HIF-1a is a downstream protein of the AMPK and regulates the expression of P-gp in MCF-7/hypoxia cells.

Moreover, the mitochondrial apoptosis related protein Bax, Cytochrome c, cleaved-Caspase 9, cleaved-Caspase 3 and cleaved-PARP do not changed after $5 \mu \mathrm{M}$ berberine alone treatment (Fig. 2C and S3), suggesting the mitochondrial apoptosis is not involved in the mechanism of DOX chemosensitivity of low dose berberine. In summary, it is demonstrated that low-dose berberine increases DOX chemosensitivity by inhibiting AMPK, subsequently down-regulation of HIF-1a and P-gp expression.
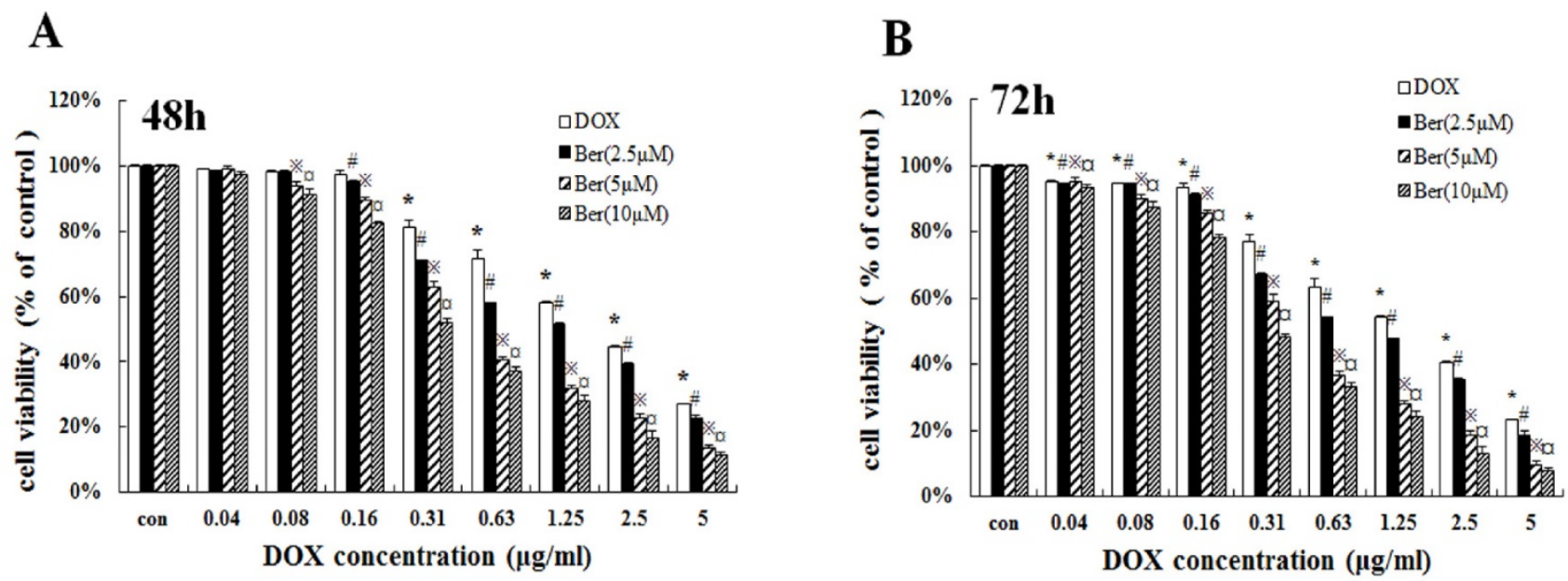

Figure 3. Low-dose berberine resensitized the DOX chemoresistance in MCF-7/hypoxial (A, B) Cell viability of low-dose berberine (2.5, 5 and $10 \mu M)$ combined with DOX in MCF-7/hypoxia cells for $48 \mathrm{~h}$ and $72 \mathrm{~h}$ treatment. Mean values \pm SD, $* \mathrm{P}<0.05$, cells treated with different concentrations of DOX vs. control group. \# $P<0.05$, cells treated with different concentrations of DOX combined with $2.5 \mu \mathrm{M}$ berberine vs. control group. $※ \mathrm{P}<0.05$, cells treated with different concentrations of DOX combined with $5 \mu \mathrm{M}$ berberine vs. control group. a $\mathrm{P}<0.05$, cells treated with different concentrations of DOX combined with $10 \mu \mathrm{M}$ berberine, vs. control group. 
A

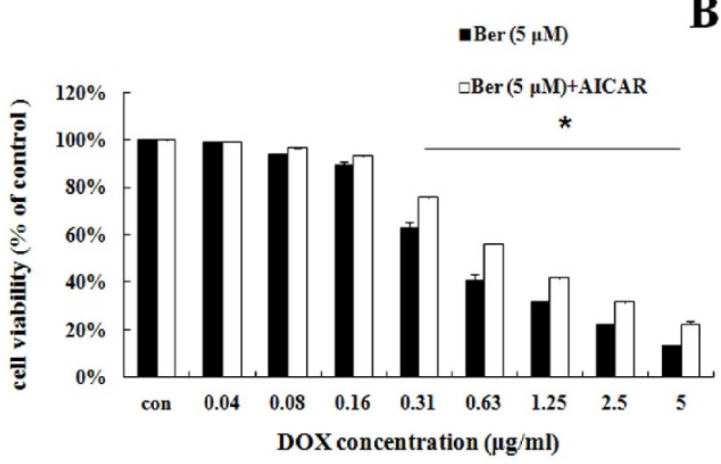

B

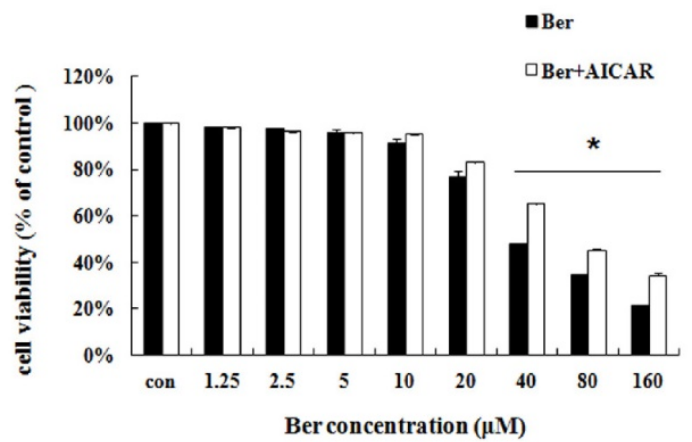

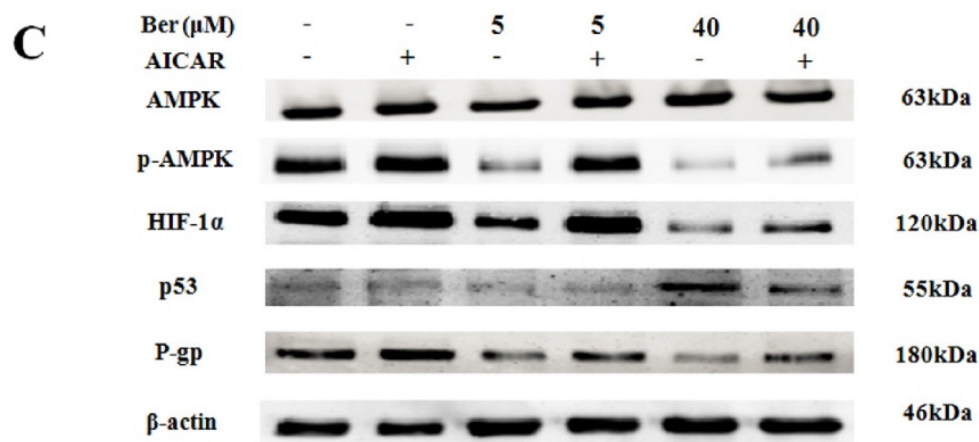

Figure 4. The effect of AMPK on berberine sensitized DOX resistance and inhibited cell growth in MCF-7/hypoxia. (A) Cell viability in MCF-7/hypoxia cells after DOX combined with $5 \mu \mathrm{M}$ berberine treatment, or DOX combined with $5 \mu \mathrm{M}$ berberine and AICAR treatment for $48 \mathrm{~h}$. Mean values \pm SD, $* \mathrm{P}<0.05$, MCF-7/hypoxia cells treated with DOX combined with $5 \mu \mathrm{M}$ berberine for $48 \mathrm{~h}$ vs. MCF-7/hypoxia cells treated with DOX combined with $5 \mu \mathrm{M}$ berberine and AICAR for $48 \mathrm{~h}$. (B) Cell viability in MCF-7/hypoxia cells after berberine treatment or berberine and AICAR treatment for $48 \mathrm{~h} . * \mathrm{P}<0.05$, MCF-7/hypoxia cells treated with different concentrations of berberine for $48 \mathrm{~h}$ vs. MCF-7/hypoxia cells treated with different concentrations of berberine and AICAR for $48 \mathrm{~h}$. (C) AMPK, HIF-1a, P-gp and p53 proteins expression after berberine treatment or berberine and AICAR treatment for $48 \mathrm{~h}$ in MCF-7/hypoxia cell. Each result was repeated three times.

A

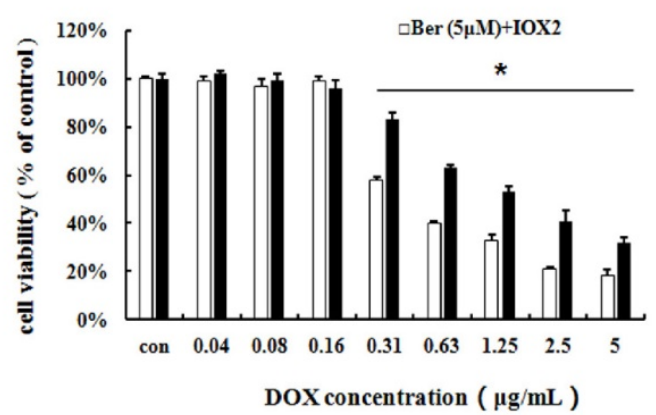

B

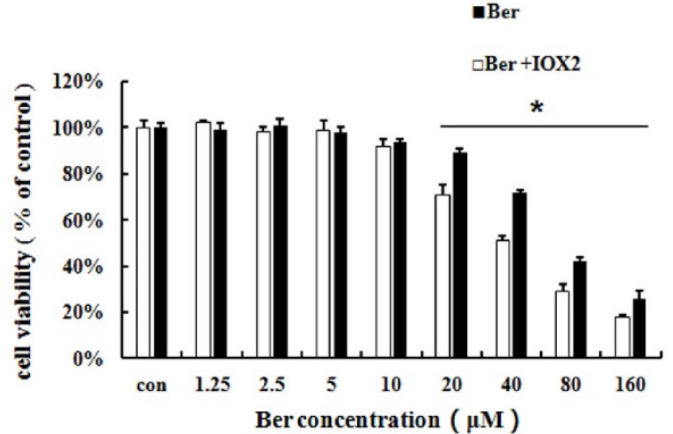

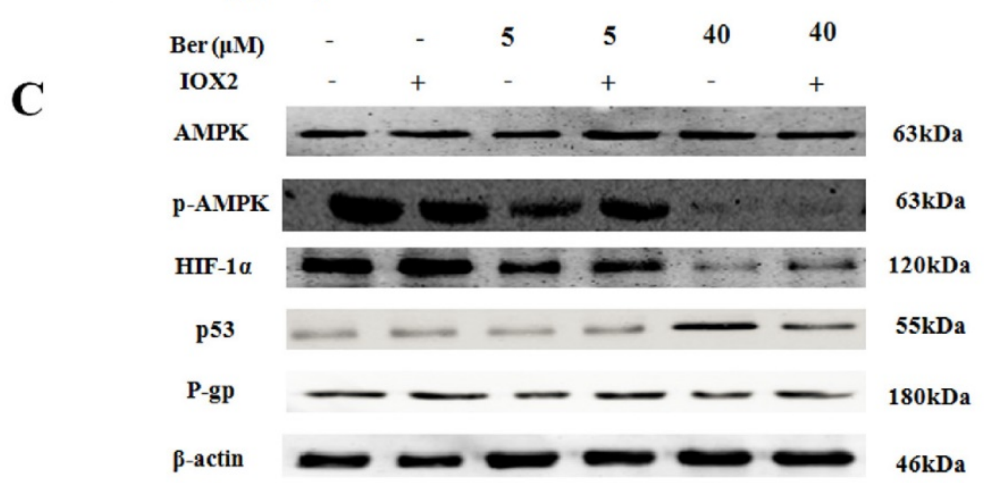

Figure 5. The effect of HIF-1 $\alpha$ on berberine sensitized DOX resistance and inhibited cell growth in MCF-7/hypoxia. (A) Cell viability in MCF-7/hypoxia cells after DOX combined with $5 \mu \mathrm{M}$ berberine treatment, or DOX combined with $5 \mu \mathrm{M}$ berberine and IOX2 treatment for $48 \mathrm{~h}$. Mean values \pm SD, $* \mathrm{P}<0.05$, MCF-7/hypoxia cells treated with DOX combined with $5 \mu \mathrm{M}$ berberine for $48 \mathrm{~h}$ vs. MCF-7/hypoxia cells treated with DOX combined with $5 \mu \mathrm{M}$ berberine and IOX2 for $48 \mathrm{~h}$. (B) Cell viability in MCF-7/hypoxia cells after berberine treatment or berberine and IOX2 treatment for $48 \mathrm{~h}$. $* \mathrm{P}<0.05$, MCF-7/hypoxia cells treated with different concentrations of berberine for $48 \mathrm{~h}$ vs. MCF-7/hypoxia cells treated with different concentrations of berberine and IOX2 for $48 \mathrm{~h}$. (C) AMPK, HIF-1 $\alpha$, P-gP and p53 proteins expression after berberine treatment or berberine and IOX2 treatment for $48 \mathrm{~h}$ in MCF-7/hypoxia cell. Each result was repeated three times. 


\section{High-dose berberine induces apoptosis by AMPK-HIF-1 $\alpha$ downregulaton inducing p53 activation}

It is showed that high-dose berberine $(40 \mu \mathrm{M})$ could directly induce apoptosis in MCF-7/hypoxia cells (Fig.2B). It is found that high-dose berberine (40 $\mu \mathrm{M}$ ) could inhibit AMPK, HIF-1a, P-gp and activate p53 (Fig. 2C and S3). To explore the downstream of AMPK signaling, AMPK activator (AICAR) and HIF-1a stabilizer IOX2 were used. As showed in Fig $4 \mathrm{~B}$, cytotoxicity of berberine (more than $40 \mu \mathrm{M}$ ) is significantly decreased by AMPK activator AICAR. As shown in Fig. 4C and S4, AICAR up-regulates high-dose berberine-induced AMPK inhibition, and high-dose berberine-induced the inhibition of HIF-1a, P-gp and activation of p53 expression are prevented by AICAR, indicating that HIF-1a,P-gp and p53 are the downstream of AMPK.

To further explore the relationship between HIF-1a and p53, HIF-1a stabilizer IOX2 was used. The cytoxicity induced by high-berberine in MCF-7/hypoxia cells is weaken by the addition of IOX2 together with down-regulation of $p 53$ protein expression, and at the same time, the p-AMPK expression does not change (Fig. 5B and 5C, S5). Our result reveals HIF-1a is a downstream protein of the AMPK and regulates the expression of p53 under hypoxia microenvironment in MCF-7 cell. Therefore, high-dose berberine promote apoptosis in drug-resistant breast cancer through AMPK-HIF-1a downregulaton inducing p53 activation.

\section{Berberine overcomes drug resistance in vivo}

The mice transplanted tumor models were used to evaluate berberine overcoming DOX resistance in hypoxia induced MCF-7 drug resistance breast cancer. Five groups were set up as Con, DOX, low dose berberine (Ber-L), low dose berberine combined with DOX (Ber-L+DOX) and high dose berberine (Ber-H). Fig. 6A-C show that tumor volumes and weights are significantly reduced in the DOX, Ber-L+DOX and Ber-H group comparing with control group, Ber-L alone group has no difference with control group and there has significant difference between Ber-L+DOX with either of Ber-L or DOX alone group, indicating that berberine increases the cytotoxicity of DOX in vivo.

Moreover, we detected the relative protein in AMPK signaling pathway in MCF-7/hypoxia xenograft. As shown in Fig. 7 and S6, low-dose berberine significantly induces the inhibition of AMPK and down-regulates the expression of HIF-1a and P-gp, while high-dose berberine promotes the expression of p53 by inhibiting AMPK- HIF-1a signaling pathway, which are consistent with the results in vitro.

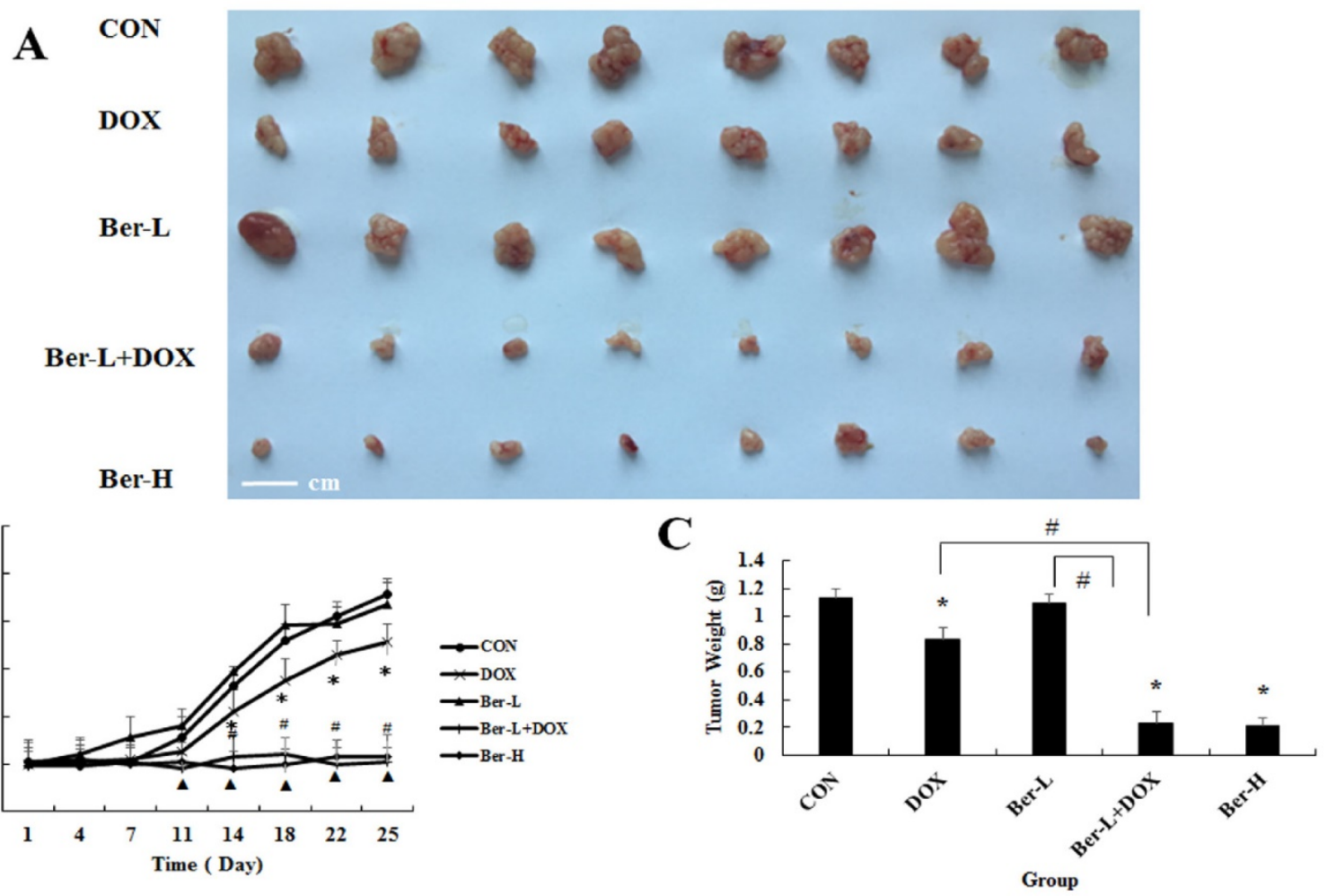

Figure 6. The overcoming hypoxia induced breast cancer drug resistance effect of berberine in vivo. (A) tumor photographs (B) tumor volume Mean values $\pm \mathrm{SD}, *$ $\mathrm{P}<0.05$, DOX vs. control group. \# $\mathrm{P}<0.05$, Ber-L+DOX vs. control group. $\wedge \mathrm{P}<0.05$, Ber-H vs. control group. (C) tumor weight $* \mathrm{P}<0.05$, mice treated with $D O X$ or (and) berberine vs. control group, \# $\mathrm{P}<0.05$ Ber-L+DOX vs. DOX or Ber-L. 


\section{CON DOX Ber-L Ber-L+DOX Ber-H}

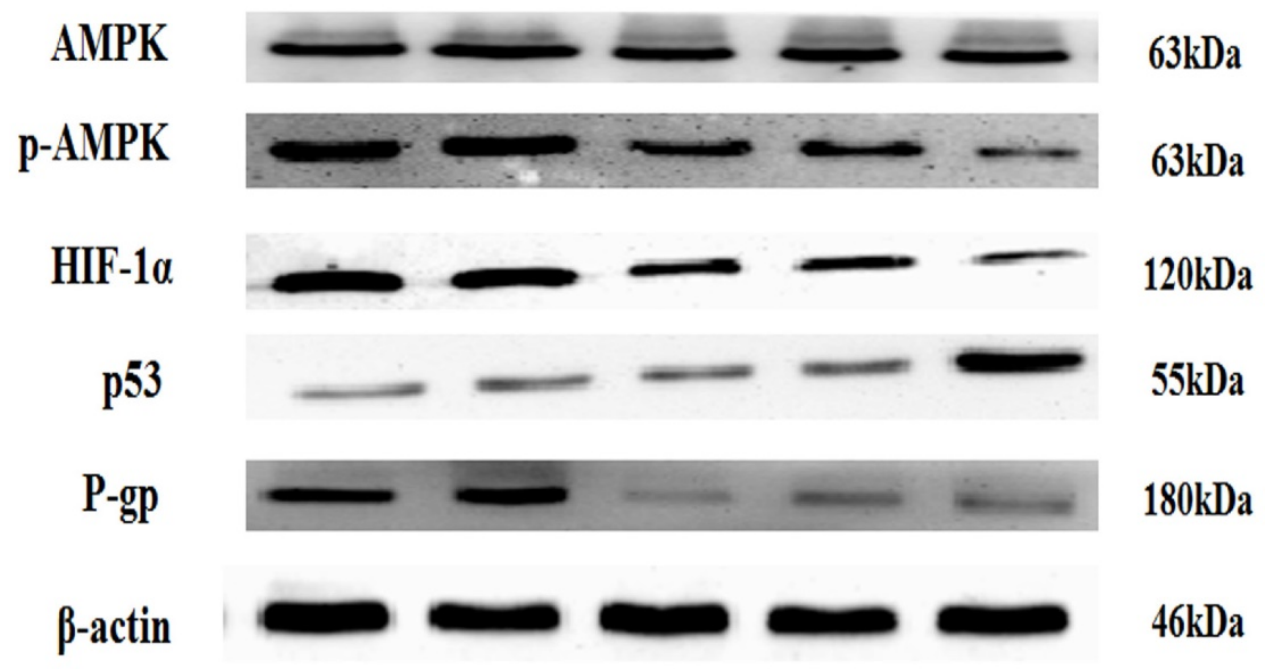

Figure 7. AMPK, p-AMPK, HIF-1a, P-gp and p53 proteins expression in MCF-7/hypoxia xenograft. Each result was repeated three times.

\section{Discussion}

Breast cancer is the most common type of cancer and the second leading cause of cancer death in American women. In 2017, 252,710 estimate new cases and 40,610 estimate deaths are projected to occur in the United States [1]. Surgery, radiation therapy, systemic treatment and personalized medicine are the main managements for breast cancer. Adjuvant chemotherapy in systemic treatment for breast cancer is popular in China, with about $81.4 \%$ of all patients with invasive breast cancer starting chemotherapy [2]. Generally, systemic agents are effective at the beginning of therapy in $90 \%$ of primary breast cancers and $50 \%$ of metastases. However, drug resistance to therapy is not only common but expected after a variable period of time. To delay the generation of chemoresistance to increase efficacy, chemosensitivity is a necessary strategy based on the breast cancer patient with drug-resistant molecular characteristics.

DOX is the most frequently used chemotherapy combined with cyclophosphamide or 5-fluorouracil. However, recent researches show that the effectiveness of DOX is considerably limited in a hypoxic microenvironment $\left(\mathrm{pO}_{2} \leq 2.5 \mathrm{mmHg}\right)$, which is typically presented in the central region of solid tumors, such as breast cancer, glioblastoma multiforme, cervical cancer, lung adenocarcinoma and hepatocellular carcinoma [19]. Hypoxia-induced MDR is one of major influencing factors on cancer therapy effectiveness [20]. AMPK is the most important cellular energy sensor which is activated by hypoxia to compensate the reduction of mitochondrial respiration. Moreover, It has been reported that AMPK activation under hypoxia induced the drug resistance in osteosarcoma (MG-63) cells [21]. In our study, we used controlled hypoxia condition to culture MCF-7 cells for about one week, the survived MCF-7 cells became hypoxia-stable and expressed higher level of AMPK than its counterpart parents. Berberine treatment enhanced the chemosensitivity of DOX to MCF-7/hypoxia in low dose and induced apoptosis in high dose, which accompanied the down-regulation of AMPK-HIF-1a. AMPK activator (AICAR) and HIF-1a stabilizer (IOX2) increase chemoresistance of DOX and apoptosis tolerance, indicating that AMPK-HIF-1a inactivation is involved in this process. It is previously reported that AMPK inhibited HIF-1a protein expression in colorectal cancer, breast cancer and gallbladder cancer cell lines under the environment of normoxia, and the canonical mechanism related to the inhibition of mTOR in translational level [22, 23]. However, we found that under the hypoxia condition, AMPK activated HIF-1a protein expression after different doses of berberine treatment. We inferred the relative mechanisms from other studies. It is probably because that the activated AMPK under hypoxia promotes the nuclear export of histone deacetylase 5 (HDAC5), which can promote the formation of more mature Hsp90-HIF-1a complex from separating less mature Hsp70-HIF-1a complex by deacetylating Hsp70, subsequently prevents the degradation of HIF-1a [24].

In the present study, we found an intriguing fact that the protein expressions of AMPK and HIF-1a were down-regulated by berberine, both low dose and high dose. But the downstream of HIF-1a occurred the bifurcation dependent on the dosage of berberine: HIF-1a-P-gp inactivation played a crucial 
role on the DOX chemosensitivity of low-dose berberine, while HIF-1a down-regulation inducing p53 activation led to apoptosis in high-dose berberine. HIF-1a is the oxygen sensor which is significantly activated in response to hypoxia [25]. Over-expressed HIF-1a is closely related chemoresistance by activating P-gp $[20,26,27]$. Consistence to these reports, our results showed that low-dose berberine could enhance DOX cheomsensitivity by inhibiting HIF-1a and P-gp expression in MCF-7/hypoxia cells. When treated with IOX2, a HIF-1a stabilizer, DOX cheomsensitivity enhanced by berberine was weaken together with the up-regulation of P-gp expression. p53 absence is associated with an increased risk of drug resistance development [28]. Our results showed high-dose berberine induced apoptosis by inhibiting HIF-1a expression and up-regulating downstream p53. IOX2 reduced the apoptosis and p53 expression after high dose berberine treatment in MCF-7/hypoxia cells. These indicated that HIF-1a plays the bridging role between AMPK and p53 in high-dose berberine-induced apoptosis under hypoxia.

In summary, this work showed that low-dose berberine could enhance DOX chemosensitivity on hypoxia-induced drug-resistant in vivo and in vitro through the AMPK-HIF-1a-P-gp pathway. High-dose berberine directly induced apoptosis on drug-resistantt breast cancer by AMPK-HIF-1a-p53 pathway. Our observations shed light on a potentially therapeutic attempt to overcome drug-resistant breast cancer.

\section{Supplementary Material}

Supplementary figures.

http://www.ijbs.com/v13p0794s1.pdf

\section{Acknowledgements}

Prof. Fengying Guan, Wenliang Liu, Fan Yao and Yang $\mathrm{Yu}$ are acknowledged for their help in preparing the paper. This work is sponsored by National Natural Science Foundation of China $(81201804,81371681)$, and the Opening Project of State Key Laboratory of Supramolecular Structure and Materials of Jilin University under Grant No. SKLSSM 201504 and 201713. Undergraduate innovative program of Jilin University (2016B79690, 2016A79311). Most of experiments were carried out at Nanomedicine Engineering Laboratory of Jilin Province and Preclinical Pharmacology R\&D Center of Jilin Province.

\section{Competing Interests}

The authors have declared that no competing interest exists.

\section{References}

1. Rebecca L. Siegel, Kimberly D. Miller, Ahmedin Jemal, Cancer Statistics, 2017. CA Cancer J Clin, 2017; 00:00-00

2. Yuan XM, Wang N, Ouyang T, et al. Current status of diagnosis and treatment of primary breast cancer in Beijing, 2008. Chin J Cancer Res, 2011; 23: 38-42.

3. Teicher BA. Hypoxia and drug resistance. Cancer Metastasis Rev, 1994; 13: 139-68.

4. Lu J, Tan M, Cai Q. The Warburg effect in tumor progression: mitochondrial oxidative metabolism as an anti-metastasis mechanism. Cancer Lett, 2015; 356: 156-64.

5. Zhihong Ai YL, Songbo Qiu, Zhen Fan. Overcoming cisplatin resistance of ovarian cancer cells by targeting HIF-1-regulated cancer metabolism. Cancer Lett, 2016.

6. Shan JZ, Xuan YY, Zhang Q et al. Ursolic acid sensitized colon cancer cells to chemotherapy under hypoxia by inhibiting MDR1 through HIF-1alpha. J Zhejiang Univ Sci B, 2016; 17: 672-82.

7. Kim HG, Hien TT, Han EH et al. Metformin inhibits P-glycoprotein expression via the NF-kappaB pathway and CRE transcriptional activity through AMPK activation. Br J Pharmacol, 2011; 162: 1096-108.

8. Rui-hua Xu HP, Yan Zhou, Jennifer S. Carew et al. Inhibition of Glycolysis in Cancer Cells: A Novel Strategy to Overcome Drug Resistance Associated with Mitochondrial Respiratory Defect and Hypoxia. Cancer Res, 2002; 65: 613-21.

9. Shrikant Pradhan DM, Prabhjot Kaur, Namita Pandey, Chandresh Sharma et al. Scriptaid overcomes hypoxia-induced cisplatin resistance in both wild-type and mutant p53 lung cancer cells. Oncotarget 2016; 7(44): 71841.

10. Erler JT, Cawthorne CJ, Williams KJ et al. Hypoxia-Mediated Down-Regulation of Bid and Bax in Tumors Occurs via Hypoxia-Inducible Factor 1-Dependent and -Independent Mechanisms and Contributes to Drug Resistance. Mol Cell Biol, 2004; 24: 2875-89.

11. Salminen A, Kaarniranta K, Kauppinen A. AMPK and HIF signaling pathways regulate both longevity and cancer growth: the good news and the bad news about survival mechanisms. Biogerontology, 2016; 17: 655-80.

12. Chang $\mathrm{W}$, Zhang M, Li J et al. Berberine attenuates ischemia-reperfusion injury via regulation of adenosine-5'-monophosphate kinase activity in both non-ischemic and ischemic areas of the rat heart. Cardiovasc Drugs Ther, 2012; 26: 467-78

13. Kim HS, Wannatung $T$, Lee $S$ et al. Quercetin enhances hypoxia-mediated apoptosis via direct inhibition of AMPK activity in HCT116 colon cancer. Apoptosis, 2012; 17: 938-49.

14. Xi Yang BY, Jing Cai, Chi Zhang et al. Berberine enhances radiosensitivity of esophageal squamous cancer by targeting HIF-1a in vitro and in vivo. Cancer Bio Ther, 2013; 14: 1068-73.

15. Su YH, Tang WC, Cheng YW et al. Targeting of multiple oncogenic signaling pathways by Hsp90 inhibitor alone or in combination with berberine for treatment of colorectal cancer. Biochim Biophys Acta, 2015; 1853: 2261-72.

16. Zhang $\mathrm{M}, \mathrm{Lv} \mathrm{X}, \mathrm{Li} \mathrm{J}$ et al. Sodium caprate augments the hypoglycemic effect of berberine via AMPK in inhibiting hepatic gluconeogenesis. Mol Cell Endocrinol, 2012; 363: 122-30.

17. Deppe J, Popp T, Egea V, et al. Impairment of hypoxia-induced HIF-1a signaling in keratinocytes and fibroblasts by sulfur mustard is counteracted by a selective PHD-2 inhibitor. Arch Toxicol, 2016; 90(5): 1141-1150.

18. Rytkönen K T, Williams T A, Renshaw G M, et al. Molecular evolution of the metazoan PHD-HIF oxygen-sensing system. Mol Biol Evol., 2011; 28(6): 1913-1926

19. Huang CC, Chia WT, Chung MF et al. An Implantable Depot That Can Generate Oxygen in Situ for Overcoming Hypoxia-Induced Resistance to Anticancer Drugs in Chemotherapy. J Am Chem Soc, 2016; 138: 5222-5.

20. Li D-W, Dong P, Wang F et al. Hypoxia Induced Multidrug Resistance of Laryngeal Cancer Cells via Hypoxia-inducible Factor-1a. Asian Pac J Cancer Pre, 2013; 14: 4853-8.

21. Zhao C, Zhang $\mathrm{Q}, \mathrm{Yu} \mathrm{T}$, et al. Hypoxia promotes drug resistance in osteosarcoma cells via activating AMP-activated protein kinase (AMPK) signaling. J Bone Oncol, 2016; 5(1): 22-29.

22. Sheng B, Liu J, Li GH. Metformin preconditioning protects Daphnia pulex from lethal hypoxic insult involving AMPK, HIF and mTOR signaling. Comp Biochem Physiol B Biochem Mol Biol, 2012; 163: 51-8.

23. Shaw R J. Glucose metabolism and cancer. Curr Opin Cell Biol, 2006; 18(6): 598-608

24. Chen S, Yin $\mathrm{C}$, Lao $\mathrm{T}$ et al. AMPK-HDAC5 pathway facilitates nuclear accumulation of HIF-1alpha and functional activation of HIF-1 by deacetylating Hsp70 in the cytosol. Cell Cycle, 2015; 14: 2520-36.

25. Qilin Ao WS, Shuang Guo, Lei Cai1, Lei Huang. SENP1 desensitizes hypoxic ovarian cancer cells to cisplatin by up-regulating HIF-1a. Sci Rep, 2015;5.

26. Lara Milane ZD, Mansoor Amiji. Role of hypoxia and glycolysis in the development of multi-drug resistance in human tumor cells and the establishment of an orthotopic multi-drug resistant tumor model in nude mice using hypoxic pre-conditioning. Cancer Cell Int, 2011;11(1):3.

27. Zhang $H$, Sun $L$, Xiao $X$ et al. Kruppel-like factor 8 contributes to hypoxia-induced MDR in gastric cancer cells. Cancer Sci, 2014; 105: 1109-15.

28. Li D, Zhou L, Huang J et al. Effect of multidrug resistance 1/P-glycoprotein on the hypoxia-induced multidrug resistance of human laryngeal cancer cells. Oncol Lett, 2016; 12: 1569-74. 